\title{
Shear flow characteristics of densely packed granular material subjected to slow deformations
}

\author{
Subodh Dhakal \\ Department of Geology, Tri-chandra Campus, Tribhuvan University, Nepal \\ (Email: subodhdhakal@hotmail.com)
}

\begin{abstract}
The densely packed assembly of granular materials subjected to slow deformations is studied experimentally in the 2D shear flow apparatus. High speed video camera and subsequent image processing techniques help to document the positions of the particles in the flow. Effective algorithms are formulated to determine the particle rotation, group size and local particle concentrations. Experimental results depict that the consecutive cycles of solid like (jammed) and fluid like (un-jammed) states characterize the flow. The jammed state is represented by negligible mobilization of particles, whereas the un-jammed state is represented by considerable mobilization of particles. The rotational and translational kinetic energy shares their dominancy in the jammed and un-jammed states respectively. Nevertheless, rotational counterpart also increases quite high in un-jammed state. There exists clearly a gradient of translational and rotational velocity across the shear cell especially in the un-jammed state indicating the phenomenon of strain localization. The un-jammed state originates because of the breaking and buckling of few columns near to the inner moving wall as noticed by previous researchers, and the jammed state regenerates once the broken and buckled columns regrouped into new columns. The dilatation phenomenon is found to be associated with the un-jamming states indicated by the drop in the local particle concentrations.
\end{abstract}

Key words: Granular shear flow, particle rotation, jammed state, un-jammed state, strain localization

Received: 1 January, 2013

Revision accepted: 14 June, 2013

\section{INTRODUCTION}

Majority of continuum mechanical models consider uniform deformation within the granular mass and the gradient in the kinematics of two adjacent particles are represented by their mean gradient. However, it is reported that the deformations in densely packed deforming granular materials are non affine (Tordesillas 2007). Therefore, micromechanics of each and every particle in the assembly of granular soil are important to know the actual deformation mechanism. In that sense, continuum approaches fall short on truly characterizing the flow properties of the dense granular flow. Owing to the difficulty on getting the particle scale information accurately in the experiment, numerical simulations and theoretical models dominate the research in this field. However, it is imperative that the results obtained from numerical simulations be validated by the experimental results. This paper attempts to characterize the flow behavior of dense granular flow by capturing the particle scale information from the experimental shear cell apparatus.

The mechanism of failure has been traditionally explained in terms of sliding of particles at contact by using different sliding models (Newland \& Alley 1957; Rowe 1962; Nemat-Nasser 1980). After Oda et al. 1982 found some strong evidences from the experimental study that rolling at the contact between particles is the major mechanism of deformation; there is growing concern over this issue in the field of granular soils. Successive studies by Oda (1997) and Oda and Kazama (1998) in the natural sand by using the $\mathrm{X}$-ray application and optical method have shown that high gradient of particle rotations exist in the shear band. Similar evidences have been observed in the numerical analysis of Iwashita and Oda (2000). In addition they found the new microstructure during the strain softening process which is something like buckling of column like structure in shear bands. This mechanism is found to be associated with particle rotation. It is reported that particle rotations have significant effects to decrease their strength and stiffness (Bardet, 1994). The numerical simulations carried out by Bardet and Huang (1992) also showed that particle rotations influence the localized failure modes of idealized granular materials. Numbers of other studies have also highlighted the phenomena of particle rotations in dense flow (Bardet 1994; Kuhn and Bagi 2004; Oda and Iwashita 2000; Dedecker et al. 
2000; Misra et. al 1997; Calvetti et. al 1997; Bagi 1993).

\section{OBJECTIVES OF STUDY}

Although, particle rotation has been considered as an important deformation mechanism of dense granular soil, the mechanism is not quite precisely understood until now. The major objective of present study is to characterize the flow pattern in densely packed assembly of granular materials with especial attention to the phenomenon of particle rotations and strain localization by using the newly designed 2D experimental shear cell. Present study is equipped to capture the particle scale information while the experiment is going on. The digital video taken by the high speed video camera and the image processing technique ensure the particle scale information for further analysis.

\section{METHODOLOGY}

\section{Experiment}

The experimental set up consists of two components: apparatus to generate shear flow and set up to capture the video for further processing of the data. The effectiveness of the methodology is increased with the application of high speed video camera instead of simple video camera used by Iwashita et al. (2004). The schematic diagram of the shear flow apparatus is shown in Fig. 1. The shear flow apparatus consists of an inner movable wall and the outer stationary wall that rest on the bottom horizontal plate (Fig. 1). The inner and outer walls both are roughened by gluing the circular flanges of same radius as the particles so that they become part of the shear flow as well. The outer wall is placed on horizontal steel plate through several ball bearings to minimize the friction between them. The velocity of inner wall can be freely controlled by changing the speed of DC Motor joined with it. The spacing between the outer and the inner wall, called shear cell, is $11.5 \mathrm{~cm}$.

The distance between the upper and the bottom plates is $8 \mathrm{~mm}$ which is slightly larger than the particle thickness $(6 \mathrm{~mm})$. The details of the apparatus and the properties of particles used in the experiments are given in Table 1 and Table 2 respectively. The apparatus is covered from the top by arcyclic plate to protect the particles from escaping away, to generate stable flow and to capture digital image while the experiment is on. Mono-size plastic discs of diameter $1.6 \mathrm{~cm}$ (Fig. 2) and with two position marks equidistant from the

(a)

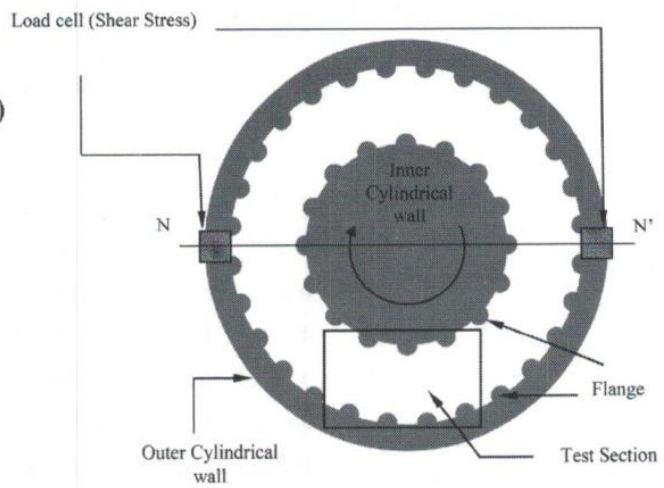

(b)

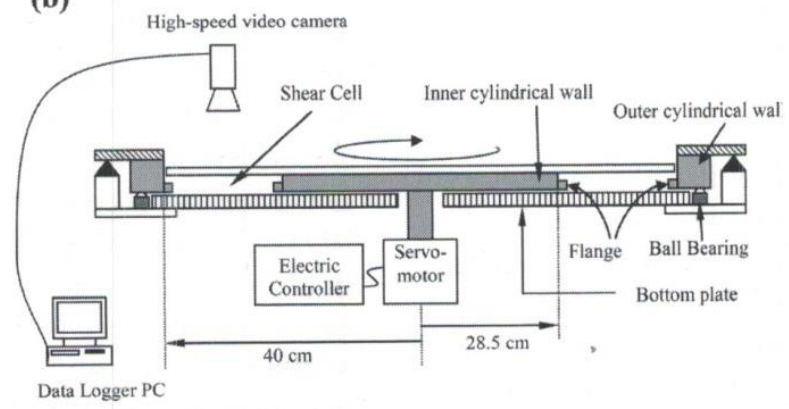

Fig. 1: The sketch of shear flow apparatus. (a) Plan view, and (b) Cross section. 
Table 1: Capacity of experimental shear cell apparatus.

\begin{tabular}{ll}
\hline Motor speed & $3 \sim 180 \mathrm{rpm}$ \\
Shear range, & $0.5 \sim 120 \mathrm{sec}-1$ \\
Maximum torque of motor & $15 \mathrm{~N}-\mathrm{m}$ \\
Radius of inner wall & $28.5 \mathrm{~cm}$ \\
Radius of outer wall & $40 \mathrm{~cm}$ \\
Diameter of flange & $2.47 \mathrm{~cm}$ \\
No. of flanges attached to inner wall & 37 \\
No. of flanges attached to outer wall & 52 \\
\hline
\end{tabular}

Table 2: Physical properties of plastic discs

\begin{tabular}{l|l}
\hline Diameter, D $(\mathrm{mm})$ & 16 \\
\hline Thickness $(\mathrm{mm})$ & 6 \\
\hline Mass $(\mathrm{g})$ & 1.127 \\
\hline Density $\left(\mathrm{g} / \mathrm{cm}^{3}\right)$ & 0.934 \\
Coefficient of restitution, e & 0.870 \\
Coefficient of friction, & 0.265 \\
\hline
\end{tabular}

center were used in the experiment so as to get the spatial co-ordinates of each of the marks. The discs are found better than the steel balls to trace the particle movement for the available experimental apparatus as suggested by Iwashita et al. (2004). Due consideration is given to reduce the friction in between particles and the shear cell. For this purpose, friction reducing silicon spray was used. The advantage of the experimental shear cell and other accessories used for this study is that particle movement can be tracked and even the collided particles and time of collision can be observed visually with reasonable accuracy.

The solid fraction $v$ is estimated as the ratio of the number of particles used in the shear cell $N_{p}$ to the maximum numbers of particles that can be accommodated $N_{\max }$ according to the geometry of the set up 'Eq. (1)'.

$$
v=\frac{N_{p}}{N_{\max }}
$$

The shear rate, $\gamma=V_{w} / H$, (defined as the inner wall velocity $V_{w}$ divided by the width of the shear cell $H$ ), is set by varying the velocity of inner wall. After introducing proper arrangement of the light system, the shear flow is recorded by the high speed video camera (FASTCAM-X 1280 PCI) which is connected with a frame grabber. The detail capacity of the high speed video camera is given in Table 3 . Each experimental run consisted of 1000 digital video frames at the precision of 60 frames per second and the resolution of 1280
512 pixels. The recorded digital video is later transferred to the particle tracking software to find the spatial positions, which will be described later.

\section{Particle tracking}

The digital video of each experiment captured by FASTCAM-X 1280 PCI is transferred to the particle tracking software called Dipp-Motion 2D produced by DITECT Co. Ltd., Japan. Each of the particles is marked at two places equidistant from the center of the particle so as to get the rotation angle between two successive time frames (Fig. 2 ). This ensures to get the center co-ordinates of the particles which are necessary to detect the contacts and to find the group size. The particle tracking software gives the coordinates of the both of the marks in each of the particles represented by black marks in Fig. 2. The basic principle of particle tracking is that it recognizes the black circular geometric shape in the image and look for the closest matching shapes to track in the next time steps. To find the maximum possible contrast between the floor of the shear cell and the particles, white colored plastic discs are used. The black circular marks in the particles give the highest possible contrast and the software can follow the same circular mark even if the particle undergoes rotation. The methodology to track the positions is such that the coordinates $\left(\mathrm{x}_{l i}, \mathrm{y}_{l i}\right)$ of the one of the marks of ith particle from frame 1 is recorded, and mapped to frame 2 where their new coordinates are recorded as $\left(\mathrm{x}_{2 i}, \mathrm{y}_{2 i}\right)$ and so on. After finishing tracking the positions of this mark for all the frames, the positions of the next mark are tracked. After finishing the tracking of this mark for all the frames, next mark is tracked in a similar fashion.

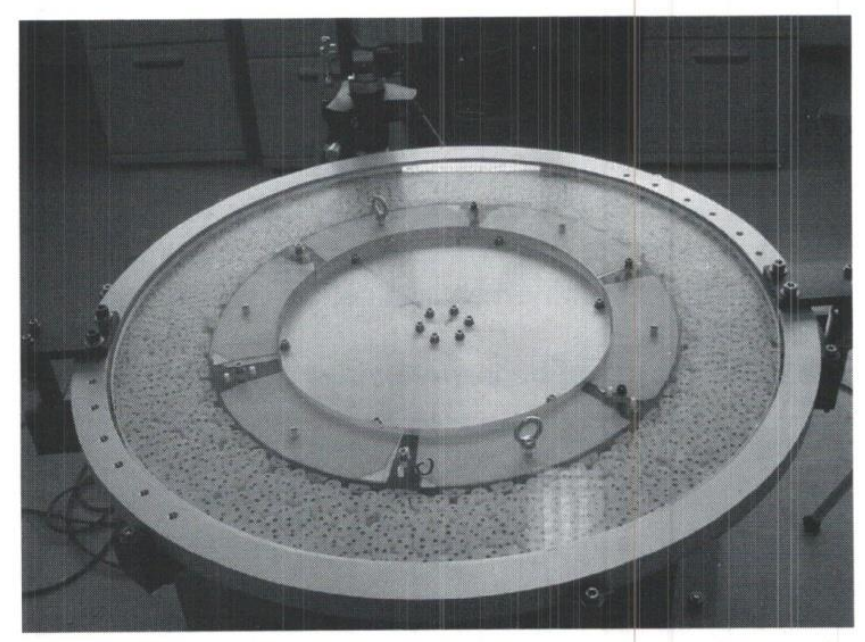

Fig. 2: Sample picture of the particles in the test section of the shear cell. The centers of particles are represented by black marks for which the spatial positions are obtained from particle tracking velocimetry. 
After necessary adjustments in the software and applying the conversion factor to get the physical co-ordinates from the pixel value as given in Table 3, the spatial coordinates of the each mark in each of the particles at each time frame were obtained. The numbers of other parameters including the velocity vectors and contact properties were determined using these spatial co-ordinates as a base data. To judge the accuracy of the tracked data, experiments were conducted using the particles that are marked at two places equidistant from the particle center and their spatial positions were obtained using particle tracking. It is found that the distance between two mark points calculated at different time frames using the position data from particle tracking method is greater than the actual distance by the maximum of $1 \mathrm{~mm}$ and minimum of $0.5 \mathrm{~mm}$, which are respectively about $5 \%$ and $3 \%$ of the particle diameter. Therefore, it is expected that the spatial position data have accuracy level greater than $95 \%$.

\section{PARAMETERS TO CHARACTERIZE}

\section{THE FLOW}

\section{Displacement and rotation of particle}

The difference in the position of the center of a particle between two successive time frames gives the displacement of that particular particle. This gives the linear distance traveled by the particle and is the direct measure of the mobilization of particles within the shear cell. However, it is equally possible that the particle travel certain distance in the angular direction or turn around its own center especially in the dense and slow flow conditions where there is limited space available to travel linearly. This is the phenomena related with angular displacement or rotation. Rotation is one of the potential mechanisms of particle kinematics in the granular flow as pointed out by some researchers but being largely underestimated till date. In this study, we give equal priority to translation and rotation so as to estimate their contribution in the movement and deformation of dense and

Table 3. Capacity of high speed video camera.

\begin{tabular}{ll}
\hline Frame rate & $60 \sim 1,000 \mathrm{~Hz}$ \\
Shutter speed & $30 \sim 10,000 \mathrm{~Hz}$ \\
Pixel resolution & $1,280 \times 512$ \\
Number of frames possible & 1000 \\
Size of the test section & $20 \times 11.5 \mathrm{~cm} 2$ \\
$\begin{array}{l}\text { Number of pixels in one par- } \\
\text { ticle }\end{array}$ & 41.4 \\
Type of color & Monochrome \\
\hline
\end{tabular}

slow granular flow.

Linear displacement can be determined from the position difference of center of particles between two successive time frames; however it is difficult to get the rotation only from the center position of particles. For this, the particles are marked at two places equidistant from their centers so as to get the center co-ordinates in the form of mid point of the two marks. The line joining the center of the particle and one of the marks of the same particle is compared between two successive time frames to find the rotation of that particular particle. The method of finding the rotation is illustrated in Fig. 3, where OR and OR' are the lines that join the center of particles and one of the marks in the particle in between two successive time frames respectively. The angle between $\mathrm{OR}$ and $\mathrm{OR}$ ' gives the rotation angle $\theta$ of particle. Same method is utilized for all the particles in the shear cell to find the rotation. The sign convention is such that the counter clockwise rotation is positive and the clockwise rotation is negative.

\section{Kinetic energy}

The kinetic energy of the particle is the energy it possesses because of its motion. The total kinetic energy of the particle can therefore be expressed as the sum of the translational kinetic energy of the center of mass and the rotational kinetic energy about the center of mass since the particles in the shear cell undergo both displacements and rotations. Translational kinetic energy ( $\left.K . E_{\text {translation }}\right)$ is due to the displacement of particle and is expressed in terms of mass $(m)$ and translational velocity $(v)$ of particles as given in 'Eq. 2 '.

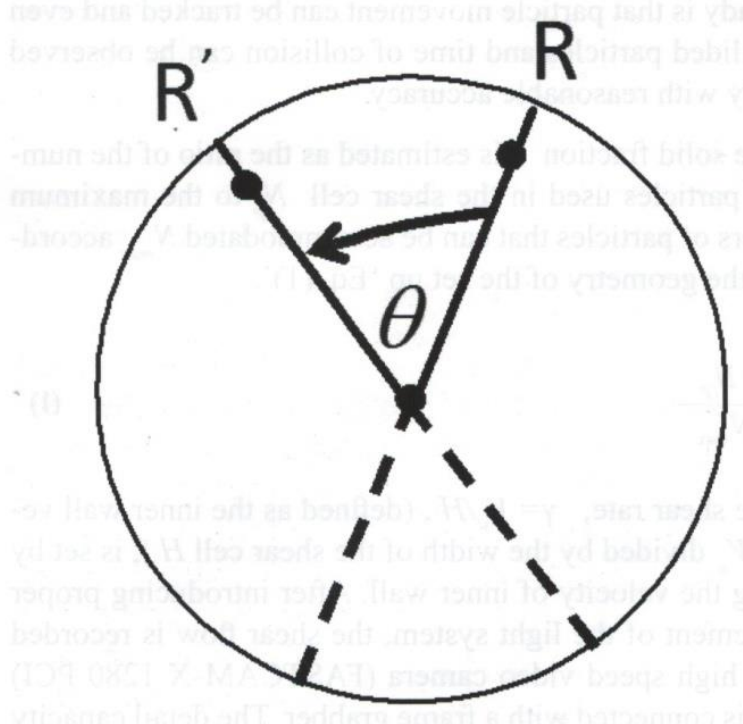

Fig. 3: Schematic diagram to illustrate the method of finding the rotation. 
The rotational kinetic energy $\left(K . E_{\text {rotation }}\right)$ is due to the rotation of the particle and is expressed in terms of the rotational velocity $(\omega)$ and the moment of inertia $(I)$ as given in 'Eq. 3'. The translational and rotational velocities are calculated by using the difference of linear and angular displacement in between two successive time frames as

$$
\begin{aligned}
& K \cdot E_{\text {rransation }}=\frac{1}{2} m v^{2} \\
& K \cdot E_{\text {rotation }}=\frac{1}{2} I \omega^{2}
\end{aligned}
$$

\section{Group size}

The group size is defined as the number of particles that are simultaneously in contact. Two distinct groups do not have any particles in contact with each other; however, all the particles that belong to the same group are connected via force networks. To determine the group size, first the contacts at each time steps were detected using the distance criteria: if the difference of the distance between two particle centers and the particle diameter is less than the threshold distance ( $5 \%$ of the particle diameter), they make a contact. All the contacts were recognized and the identities of collision partners were recorded. After counting the particles directly in contact with particle $\mathrm{i}$ and assigning a group name (such as I) for this collection of particles, other particles not directly in contact with particle i but in contact with any members of group I are counted and added to the same group. This count defines the size of that particular group. The same method of counting was iterated for all remaining particles that were not in contact with particle $i$ or any members of group I. If no particles are found in contact with particle $i$, it is skipped and the next particle is considered. In this way, some particles can be found to be isolated and not part of any group, and others can make distinct groups with a number of particles in each group. The total number of particles involved in making all the groups in a particular time step divided by the number of groups in that particular time step gives the average group size.

\section{Local solid fraction}

The initial solid fraction $v$ of the total flow is estimated as the ratio of the number of particles used in the shear cell $N_{p}$ to the maximum numbers of particles that can be accommodated $N_{\max }$ as given by 'Eq. (1)'. However because of the inhomogeneous nature of the flow, the local solid fraction in the test section of the shear flow could differ from that of the initial solid fraction and could also vary time step by time step. Therefore, measurement of local solid fraction in each time step can be fruitful. To calculate the local solid fraction, number of particles that could be accommodated in the test section of the shear cell is first estimated and the total numbers of particles that are found in each time steps are determined from the digital video with the help of particle tracking software. The ratio of the numbers of particles found in the test section of the shear cell, $N_{p(t e s t)}$, in each time step to the maximum numbers of particles that could be accommodated in it, $N_{\max (t e s t)}$, gives the local solid fraction, $v_{\text {local }}$ in the respective time frames as shown in 'Eq. (4)'.

$$
v_{\text {local }}=\frac{N_{\text {pltest })}}{N_{\max (\text { lest })}}
$$

\section{EXPERIMENTAL RESULTS}

\section{Global characteristics}

The global characteristics represent overall scenario of shear flow under investigation. As pointed out earlier, the main focus of this study is the densely packed granular shear flow under slow deformations conditions. In this regime of shear flow, it is observed that consecutive cycles of near solid behavior (jamming) and fluid behavior (un-jamming) characterize the flow. Each un-jammed state is represented by the high rotation and translational velocities compared to the mean as shown by the distinct peaks in Fig. 4, whereas each jammed state is represented by negligible rotational and translational velocities as shown by almost flat curves

(a)

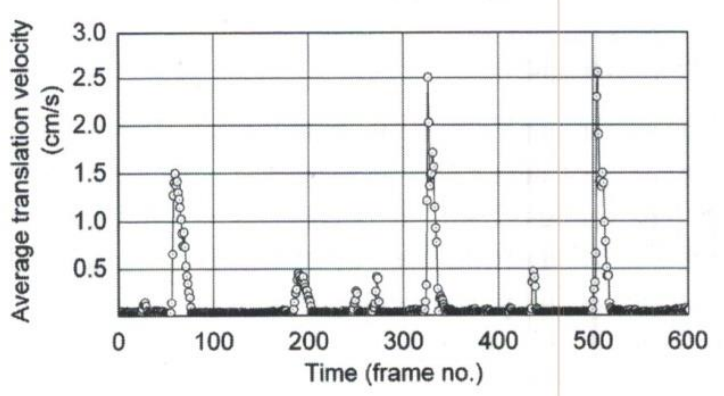

(b)

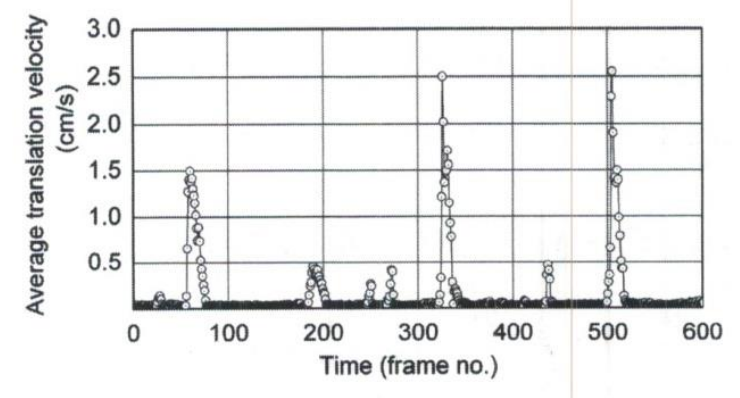

Fig. 4: Temporal variation of (a) translational velocity (b) rotational velocity. 


\section{Subodh Dhakal}

in the same Fig.. Each of the cycles continues for some time but the jammed state is in general long lasting compared to the un-jammed one.

\section{Local characteristics}

One complete cycle of jammed and un-jammed state represent the local flow characteristics. It is seen that the change of state from jammed to un-jammed conditions is a gradual process and it takes some time to complete the un-jamming event. It is important to note that the rotational velocity is considerable compared to the translational one in the jammed state as evident by the comparison of the two curves shown in Fig. 5. In this state the total energy of the system is taken over by the rotational kinetic energy which can be observed if the proportion of the translational and rotational kinetic energy is compared. The translational kinetic energy dominates the total energy of the system in the un-jamming state, nevertheless the rotational kinetic energy also increased tremendously in this state. It is reasonable to consider that the rotational kinetic energy in the jammed state is changed to translational kinetic energy in the unjamming state. The average group size and the number of groups in the flow remain constant for the whole period of jammed state. As the un-jamming state starts taking place, the average group size starts decreasing whereas the number of groups in the flow starts increasing signifying that there is mobilization of contacts in this transition (see Fig. 6).

\section{Strain Localization}

The strain localization into narrow shear bands during the deformation of dense granular materials has been described in the past studies (Tordesillas 2007; Oda and Kazama 1998; Iwashita and Oda 2000; Bardet 1994; Rechenmacher 2006). In the present study, the profile of velocity and kinetic ener-

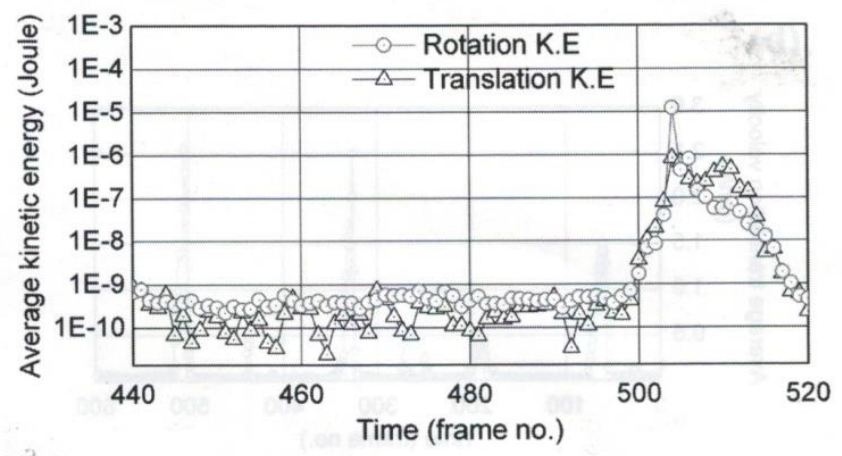

Fig. 5: Variation of rotational and translational kinetic energies in one complete cycle of jamming and unjamming events.

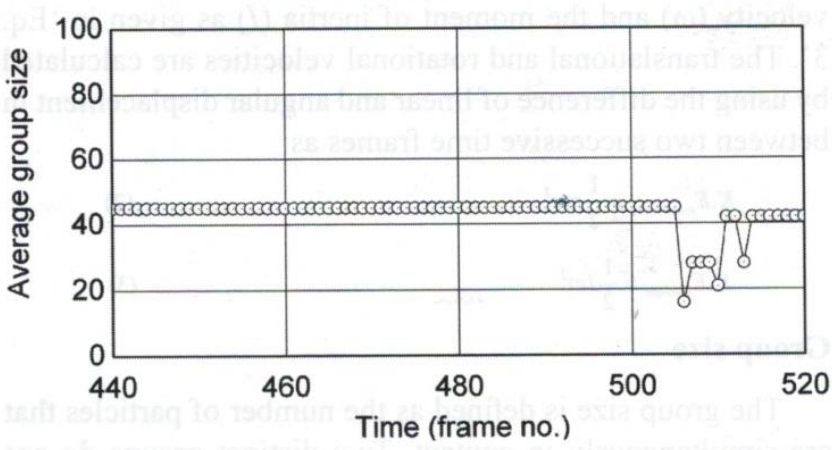

Fig. 6: Temporal variation of average group size in the jamming to un-jamming transition (shear rate 1.2/s).

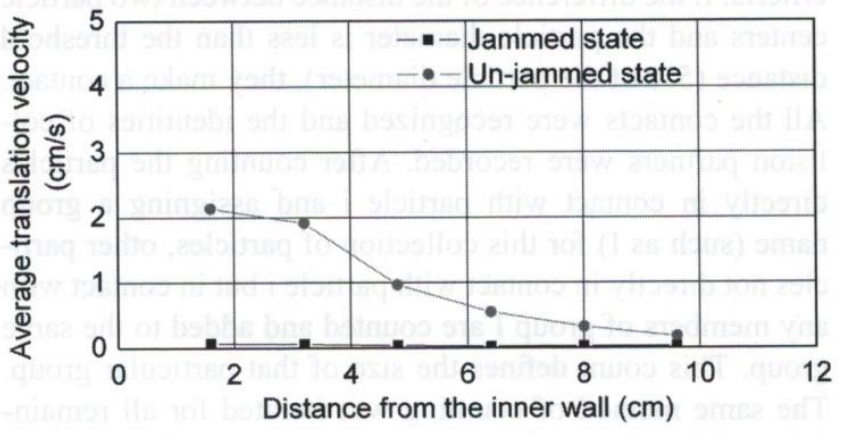

Fig. 7: Variation of average translational velocity across the shear cell.

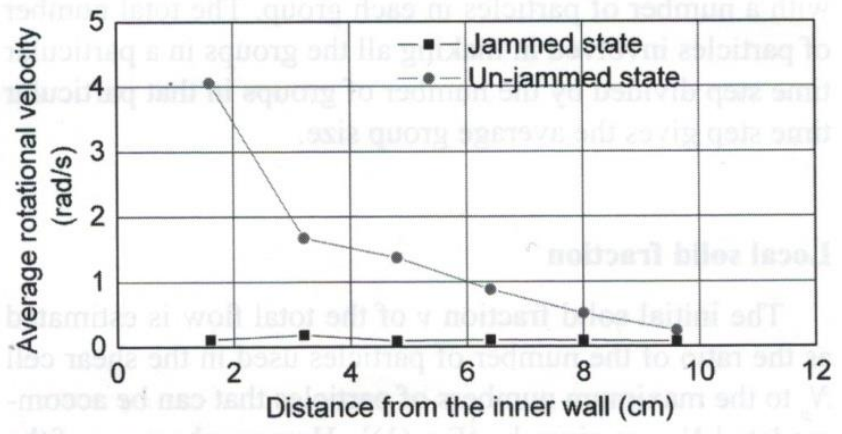

Fig. 8: Variation of average rotational velocity across the shear cell.

gy of the system of granular flow across the shear cell is considered to check this phenomenon. The profiles start from very near to the inner moving wall and ends at very near to the outer stationary wall (far from the inner wall). Both the translational and rotational velocity profile shown respec- 
tively in Fig. 7 and Fig. 8 in the un-jamming states depict that the particles are mobilized mostly very near to the inner wall and the particles near to the outer wall almost don't move even in the un-jamming state. Therefore there exist a clear gradient of velocity profile across the shear cell. This narrow band of energy localization resembles the shear band as described by previous researchers. This is the area where the displacements and rotations concentrate and is responsible for the initiation of un-jammed state from jammed state.

\section{DISCUSSIONS}

In this study, it is found that the flow is characterized by the consecutive cycles of near solid (jammed) state and near fluid (un-jammed state). Similar cycles of jammed and un-jammed states were observed by Tordesillas (Tordesillas 2007 ) in the numerical simulations. The strain localization phenomenon as observed by Oda 1997; Oda and Kazama 1998; Bardet 1994 among others is also observed in this study. The particles near to the moving inner wall possess high kinetic energy whereas far from the inner wall it diminishes. The maximum width of this energy localization zone is abou: four particle diameters. One of the important

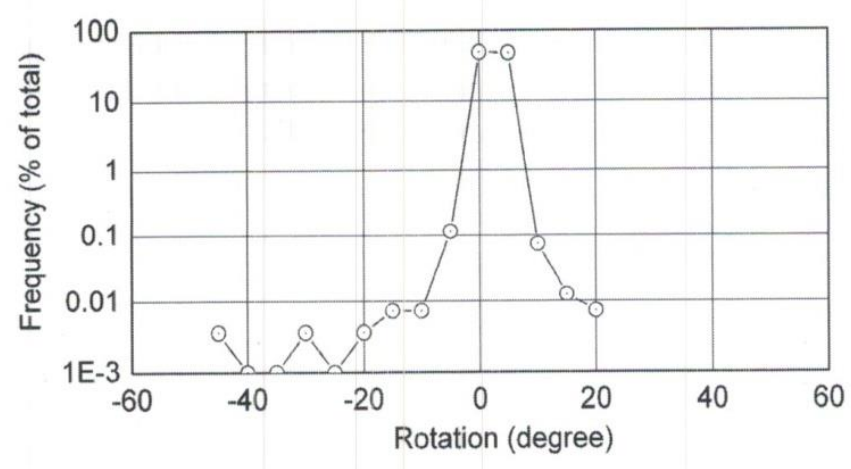

Fig. 9: Frequency distribution of rotation of particle.

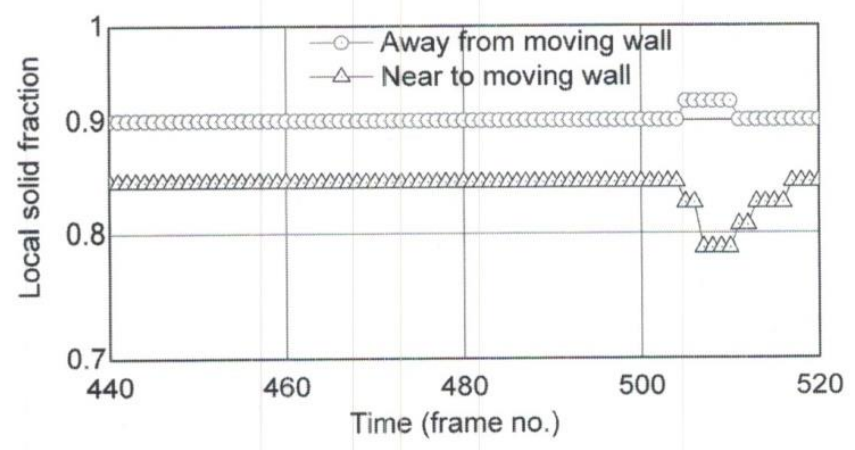

Fig. 10: Temporal variation of local solid fraction across the shear cell. The flat curves represent the jammed state and the peaks represent un-jammed state. observations in this study is that particle rotation is considerably high in magnitude in the un-jamming state especially very near to the inner wall (strain localization zone). Some of the particles rotate by even more than 40 degrees (Fig. 9).

The high degree of particle rotation seen in Fig. 9 is particularly found in un-jammed state. It is also important to note that in the un-jamming state, the translational displacement of particles also accelerated high in the shear band because of the conversion of rotational kinetic energy stored during jammed state to translational one. This is possible because of the breaking of the columns of particles which are formed in the jammed state due to the compression of particles. The phenomenon of breaking of columns is also supported by the decrease in average group size and increase in numbers of groups in the un-jamming state. Further, the local solid fraction in the test section of the shear cell decreases in the un-jamming state (Fig. 10) which support the phenomenon of dilatation as observed by previous researchers (Oda 1997; Oda and Kazama 1998; Bardet 1994).

\section{CONCLUSIONS}

The dense and slow regime of granular flow is characterized by the consecutive cycles of near solid state (jammed state) and near fluid state (un-jammed state). The jammed state is the result of the formation of condensed columns and the prolonged contacts with each other. The un-jammed state originates when some of the column breaks because of the energy localization in narrow shear band very close to the inner moving wall. The shear band is the area where most of the translational and rotational velocities concentrate. The rotational kinetic energy dominates the jammed state whereas un-jammed state is characterized by dominancy of translational velocity. When the flow convert from jammed to un-jammed state the rotational energy carried by the particles in the shear band is transformed to the translational energy. Reverse process occurs when the flow changed from un-jammed to jammed state. In that sense, translation and rotation of particles go side by side in the densely packed and slowly deforming granular soil. Further, particles in the shear band rotates by as high as 40 degrees which can have influencing role in the deformation mechanism of densely packed slowly deforming shear flow.

\section{ACKNOWLEDGEMENTS}

The financial support to the author is provided by Ministry of Education, Science, Sports and culture, Japan. This research was partially supported by the Japan Society for the Promotion of Science (JSPS), Grant-in-Aid for Scientific Research (C), Grant Number 18560482, 2006. 


\section{REFERENCES}

Tordesillas, A., 2007, Force chain buckling, unjamming transitions and shear banding in dense granular assemblies, Philosophical Magazine, v. 87, No. 32, pp. 4987-5016.

Newland, P. L.\& Alley, B. H., 1957, Volume changes in drained triaxial tests on granular materials, Geotechnique, v. 7, pp. 1734.

Rowe, P. W., 1962, The stress dilatancy relation for static equilibrium of an assembly of particles in contact, Proc. Roy. Soc. A v. 269 , pp. $500-527$.

Nemat-Nasser, S., 1980, On behavior of granular materials in simple shear, Soils and Foundations, v. 20, No. 3, pp. 59-73.

Oda, M., Konishi, J. and Nemat-Nasser, S., 1982, Experimental micromechanical evaluation of strength of granular materials, effect of particle rolling, Mech. Mater 1, pp. 267-283.

Oda, M., 1997, A micro-deformation model for dilatancy of granular materials. In: Chang, C. S et al., editors, Symposium on mechanics of particulate materials in $\mathrm{McNu}$ conference, ASCE; pp. 24-37.

Oda, M. and Kazama, H., 1998, Microstructure of shear bands and its relation to the mechanisms of dilatancy and failure of dense granular soils, Geotechnique 48, No. 4, pp .465-481.

Iwashita, K. and Oda, M., 2000, Micro-deformation mechanism of shear banding process based on modified distinct element method, Powder Technology, v. 109, pp. 192-205.

Bardet, J. P., 1994, Observations on the effects of particle rotations on the failure of idealized granular materials, Mechanics of Materials 18, pp. 159-182.

Bardet, J. P. and Huang, Q., 1992, Numerical modeling of micropolar effects in idealized granular materials, Mechanics of
Granular Materials and Powder Systems, v. 37, ASME, pp.8592.

Kuhn, M. R. and Bagi, K., 2004, Contact rolling and deformation in granular media, International Journal of Solids and Structures, v. 41, pp. 5793-5820.

Oda, M. and Iwashita, K., 2000, Study on couple stress and shear band development in granular media based on numerical simulation analyses, International Journal of Engineering Science, v. 38, pp. 1713-1740.

Dedecker, F., Chaze, M., Dubujet, P. and Cambou, B., 2000, Specific features of strain in granular materials, Mechanics of Cohesive-Frictional Matter., v. 5(3), pp. 173-179.

Misra, A. and Jiang, H., 1997, Measured kinematic fields in the biaxial shear of granular Materials, Computers and Geotechnics, v. 20 (3), pp. $267-285$.

Calvetti, F., Combe, G. and Lanier, J., 1997, Experimental micromechanical analysis of a 2D granular material: relation between structure evolution and loading path, Mechanics of Cohesive-Frictional Mater, v. 2 (2), pp. 121-163.

Bagi, K., 1993, On the definition of stress and strain in granular assemblies through the relation between micro- and macro-level characteristics, Powders \& Grains 93, C. Thornton, ed., A.A. Balkema, Rotterdam, pp. 117-121.

Iwashita, K., Ichiba, K., Oda, M., 2004, Observations of rapidly sheared granular materials, J. JSCE, v. 764/III-67, pp. 147156.

Rechenmacher, A. L., 2006, Grain-scale processes governing shear band initiation and evolution in sands, Journal of the Mechanics and Physics of Solids, v. 54, pp. 22-45. 